\title{
Calculation of involved energies in thermal plasma at the opening of high voltage breakers
}

\author{
A. Ziani ${ }^{1}$, H. Moulai ${ }^{1}$, A. Nacer ${ }^{1}$ \\ ${ }^{1}$ Laboratory of Electrical and Industrial Systems, \\ FEI, USTHB,BP 32 Bab Ezzouar, Algiers, 16311, Algeria.
}

Phone/Fax number:+ Tel. 00213 771415065, e-mail: ziani08@yahoo.fr

\begin{abstract}
.
Usually, calculations of involved energies in thermal plasma at the opening of high voltage breakers are led by using dimensional type models (1D, 2D and 3D) founded on assumptions sufficiently precise. This survey deals with the determination of temperature and thermal transfers of arc plasma quenching in a high voltage breaker by coupling a OD (time dependent) arc model with the heat equation. This approach enabled to refine the thermal balance and to evidence the particular importance of the thermal radiation. Also, investigations are focused on the influence of the deionisation constant of the extinguisher gas on the plasma conductance and temperature variations which are checking parameters of the extinction. The obtained results are in good agreement with measurements performed by other authors
\end{abstract}

Key words: Electric arc, High voltage, Arc plasma

\section{Introduction}

Thermal exchanges in high voltage breakers plasmas have been the subject of numerous dimensional models development (1D, 2D, 3D) achieved on complex geometries where few justifications are made as for the limit conditions than for the meshing $[6,9$, 13, 14, 15]. These models are more useful for breakers design phase, whereas their operating control needs for time dependent data. Models have been proposed for that purpose but remain enough approximated as they don't take in account all the involved forms of energy, especially the thermal radiation, during the extinction phase of the arc $[3,5]$. Currently, most authors integrate the radiation effect in thermal plasmas by using the net emission coefficients introduced by Liebermann and Lowke [17] with questionable and less realist hypotheses (isotherm plasma of cylindrical or spherical geometry).

This article intends to introduce a new theoretical approach to follow the thermal transfer evolvement during the extinction of $\mathrm{SF}_{6}$ arc plasma by coupling a OD thermal model that takes in account the thermal radiation and the heat equation. Three practical values of the deionization constant have been considered [1, $3,10]$.

The three forms of thermal transfer have been taken in account to build up an energy balance for $\mathrm{SF}_{6}$ arc plasmas applied to a high voltage line breakers $(245 \mathrm{kV} / 50 \mathrm{kA} / 50 \mathrm{~Hz})$ which has been the subject of several experimental studies $[3,5]$.

\section{Formulation of the Problem}

The heat equation can be then written:

$\rho c_{p} \frac{\partial T}{\partial t}=\frac{1}{g} i^{2}+h\left(T_{e x}-T\right)-\operatorname{div}\left(-K_{T} \operatorname{gra} d(T)\right)-P_{R a y}(1)$

Where $T$ is temperature, $\rho$ the density, $c_{p}$ the massic heat at constant pressure, $h$ the coefficient of thermal exchange, $K_{T}$ the thermal conductivity, $P_{\text {Ray }}$ the power lost by radiation and $i$ the arc current. At the opening of the contacts, an electric arc will appear and generate an arc voltage $u_{\text {arc }}$ that will condition the energy $W_{1}$ dissipated by Joule effect in the circuit breaker, implying thus a very high increase of temperature. It is given by the relation: $W_{1}=\int_{t_{0}}^{t_{f}} u_{\text {arc }} i d t$; where $i$ is the current in the arc column, t0 and $t_{f}$ are respectively the times of the beginning and the end of the breaking. The expression of the convection energy $d W_{2}$ evacuated during an elementary interval of time $d t$ is given by: $d W_{2}=-h\left(T-T_{0}\right) d t$

The assessment of the exchange coefficient $h$ is enough complicated because of its dependence on temperature. Xiang Zhang et al [4] use a value of $h$ for $\mathrm{SF}_{6}$ close to the one of air $\left(10 \mathrm{Wm}^{-2} \mathrm{~K}^{-1}\right.$ [4]). An approached value of the emitted power is obtained by the calculation of the energy balance of this phenomenon described quantitatively by Stephan Boltzmann's equation. 
We can therefore determine the energy $d W_{3}$ radiated per surface unit by the plasma during an elementary time $d t: d W_{3}=-\varepsilon \sigma\left(T^{4}-T_{0}^{4}\right) \cdot d t$

Where $\mathcal{E}$ represents the emissivity of the plasma which varies between 0 and 1 , and $\sigma$ the coefficient of Stefan Boltzman ( $\sigma=5.6810^{-8} \mathrm{Wm}^{-2} \mathrm{~K}^{-4}$ ).

A new OD approach of arc quenching has been previously developed [7] and implicates the thermal radiation emitted by the electric arc during its extinction. This approach yields good results especially at the beginning of breaking. A new equation that models the arc extinction at the opening of the poles of the breaker has been then obtained:

$$
\frac{d \ln g}{d t}=\frac{1}{\tau}\left(\frac{u^{2}}{u_{a}^{2}}-1-\frac{\sigma \varepsilon\left(T^{4}-T_{0}^{4}\right)}{g u_{a}^{2}}\right)
$$

For this calculation, we consider an arc of cylindrical symmetry in local thermodynamic equilibrium. The pressure is assumed to be equal to 1 Atmosphere during the arc extinction and the thermodynamic coefficients are constants. One also considers that the electric field remains constant inside the arc column.

The equation obtained in the above model has been coupled with the equations of the heat, the thermal transfer by conduction, the power of radiation and the energy lost by Joule effect. One obtains then a system of 5 differential equations with 5 variables: $g, T, W_{l}$, $W_{2}$, and $W_{3}$ that will be solved numerically by Runge Kutta method for a $90 \%$ default current.

\section{Results of simulation}

The necessary initial conditions to the numerical solving are as: $W_{1}=W_{2}=W_{3}=0 ; T(0)=10000$ $\mathrm{K} ; \mathrm{g}(0)=10^{4} \mathrm{Sm}^{-1}$. The thermal exchange factor $h$ is taken, in the case of a forced puffer circuit breaker, equal to $10 \mathrm{Wm}^{-2} \mathrm{~K}^{-1}$.

The density and calorific capacity of $\mathrm{SF}_{6}$ have been taken from the results of $\mathrm{P}$. Chévrier et al [14] at a pressure of $0.1 \mathrm{MPa}$ with $\rho=3 \mathrm{kgm}^{-3}$ and $c_{p}=$ $1.5 \mathrm{Jkg}^{-1} \mathrm{~K}^{-1}$. We also consider the plasma as a grey body of emissivity $\mathcal{E}=0.9$, and the thermal exchange by conduction will be neglected ( $K_{T}=0$ ).

The source element is the power emitted by the arc; it is expressed by : $i^{2} / g$. The numerical solving of the arc equations is executed using well adapted parameters: $u_{a}=3.8 \mathrm{kV}[3,7]$, temperature between $10 \mathrm{kK}$ and $17 \mathrm{kK}[1,8,15]$ and duration of the simulation between 0 and 90 microseconds in order to avoid the Transient Recovery Voltage (TRV) [1].
Most of the works consecrated to arcing in high voltage breakers neglect the phenomenon of cooling by convection with regard to conduction $[1,2,6,7$, $10,12]$. It is as well as in this paper, the investigations will be focused on thermal convection effect.

Figure 1 represents the calculated evolution of the arc voltage and current at the opening of high voltage breakers. Figure 2 describes the temperature evolution for the three considered values of $h$. One can also notice the existence of two phases (thermal expansion - cooling). The decrease of the arc temperature is obtained rapidly for $\tau=0.27 \mu$ s. The maximum of temperature is reached after $58 \mu \mathrm{s}$ for $\tau=0.5 \mu \mathrm{s}$ and after $38 \mu$ s for $\tau=0.4 \mu \mathrm{s}$. let be a delay of $20 \mu \mathrm{s}$.

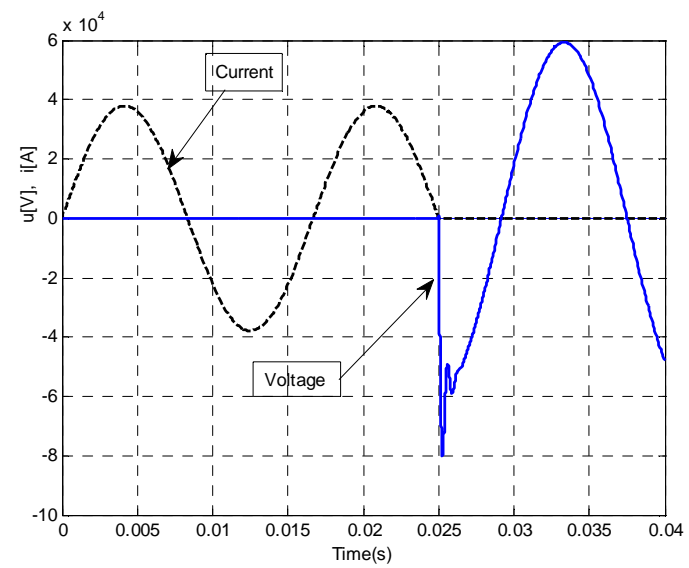

Figure1. Arc voltage and current

In such arcs, cooling processes (convection and radiation) are dominant with regard to the calorific effect. These results agree well with those reported by J.J. Lowke et al [14] that foresee a very fast decrease of the conductance during the $100 \mu$ s of the breaking. On figure 2 , the deionization constant $\tau$ has been set to $0.27 \mu \mathrm{s}$. Then, one first observes a fast arc temperature rise between 0 and $30 \mu \mathrm{s}$, passing from $17 \mathrm{kK}$ to $20 \mathrm{kK}$ for an initial temperature of $17 \mathrm{kK}$, afterwards, for times greater than $30 \mu \mathrm{s}$ (i.e. for currents lower than 1300A), the arc gets cold.

Two phases are therefore visible on this figure: a thermal expansion phase owed to the Joule effect injection in the arc column and a cooling phase linked to $\mathrm{SF}_{6}$ blowing. This evolution in two phases has also been reported by Rachard et al. [9]. J. C. Lee et al. $[11,12]$ also find again a succession of a thermal expansion phase followed by an extinction phase.

Cooling velocities between $0.35 \mathrm{kK} / \mu \mathrm{s}$ and $0.5 \mathrm{kK} / \mu \mathrm{s}$ have been obtained. These values are close to the ones obtained by J. B. Belhaouari et al. [9] which have modeled the arc temperature in 1D geometry while basing on Elenbass-Heller's equation [1]. 


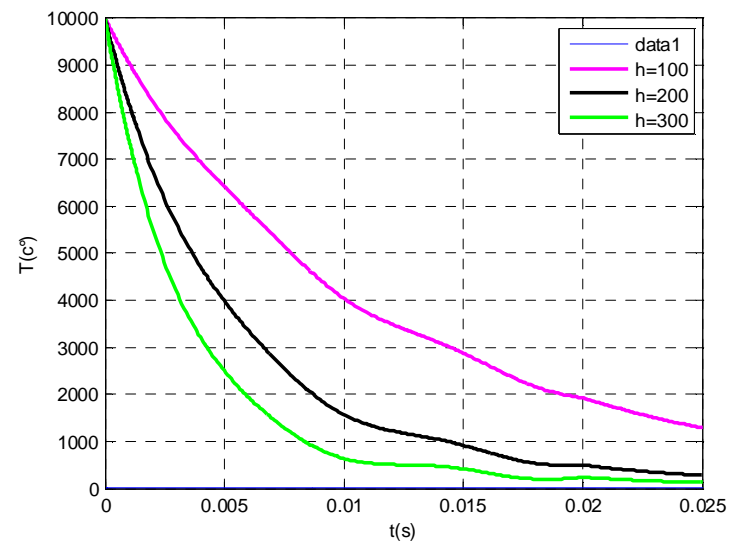

Fig.2.Temperature evolution for $\tau=0.27$ and $\mathrm{T}=10 \mathrm{kK}$

Results of thermal exchange simulation are presented on figure 3 for the 3 considered values of the deionization constant. The plasma is assumed as a gray body [7] and the emission is calculated by the means of the emissivity factor $\varepsilon$. Thermal transfers are drawn on figure 3 for $0.27 \mu$ s and a thermal exchange coefficient $\mathrm{h}=10 \mathrm{Wm}^{-2} \mathrm{~K}^{-1}$. The generation of the arc implicates a thermal transfer increase.

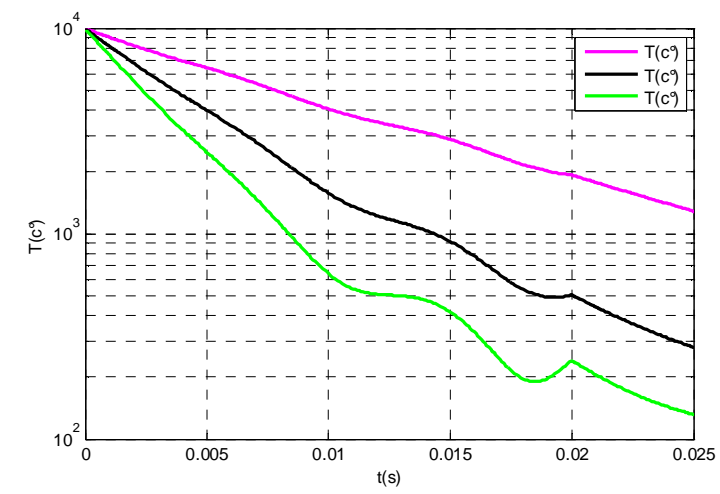

Figure 3. Temperature evolution for $\tau=0.27$ and $\mathrm{h}=10 \mathrm{Wm}^{-2} \mathrm{~K}^{-1}$

The calorific energy injected in the arc reached rapidly $120 \mathrm{~kJ}$. This thermal expansion is owed to very high densities of the arc current. This phenomenon has been observed by Xiang Zhang et al [4]. One can note that the convection and radiation energies are greater than the calorific energy, meaning that the arc is in extinction. Curves $\mathrm{a}, \mathrm{b}$ and $\mathrm{c}$ of figure 4 evidenced that the radiation energy is the term that dominates the thermal exchanges of the arc. This observation agrees well with the results obtained by Rachard et al. [7] in bi dimensional simulation.
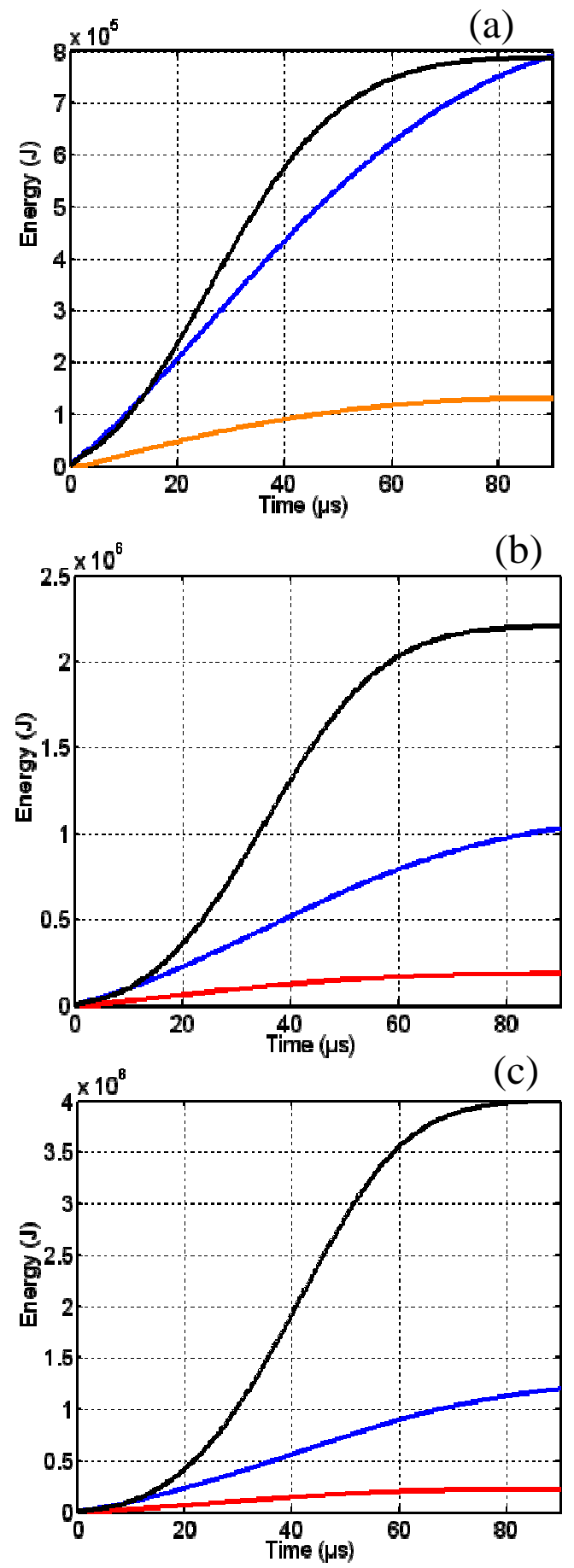

Figure 4. Thermal transfers evolution for (a): $\tau$ $=0.27 \mu \mathrm{s},(\mathrm{b}): \tau=0.4 \mu \mathrm{s}$ and $(\mathrm{c}): \tau=0.5 \mu \mathrm{s}$.

\section{Conclusion}

Two phases of arc evolution have then been evidenced. The first, during which the temperature increases under the effect of the strong thermal expansion, followed by the second phase where one observes a decrease of temperature which is linked to the arc quenching. However, radiation effect has been overestimated with regard to convection as this work is solely based on the laminar regime, neglecting then the turbulent phenomena in the plasma that are important in the cooling process. 


\section{References}

[1] S. Vacquié, Arc électrique, Techniques de l'Ingénieur, Traité de Génie Electrique D 2870, 1995.

[2] CIGRE Working Group 13-01, Applications of Black Box Modelling to Circuit Breaker, Electra, 149, 41-71, 1993

[3] L. Reynard, Modélisation tridimensionnelle de l'arc électrique dans un disjoncteur basse tension, doctorat thesis, Ecole centrale de Lyon, France, 2006.

[4] A. Ziani and H. Moulai, "Extinction properties of electric arcs in high voltage circuit breakers", Journal of Physics D: Applied Physics, Vol. 42, No 10, 2009.

[5] A. Ziani and H. Moulai, 'OD Model of thermal exchanges at the opening of an SF6 high voltage circuit breaker", ACTA Press, Proc. of the Int. Conf. on Power and Energy systems, IASTED Europe's 2009, 07-09 September 2009, Palma de Mallorca, Spain.

[6] C. Fiévet, C. Fleurier and D. Hong, ICPIG XXI, Bochum (1995).

[7] H. Rachard, P. Chévrier, D. Henry, and D. Jeandel, Int. J. Heat and Mass Transfer 42, 1723 (1999).

[8] M. Ushio and F. Matsuda, JWRI 11, 7 (1982).

[9] J.B. Belhaouari, Doctorat Thesis, Paul Sabatier University, Toulouse, France, N`2780 (1997).

[10] J. C. Lee and Y. J. Kim, Surface and Coatings Technology 201, 5641 (2007).

[11] J. C. Lee and Y. J. Kim, Thin Solid Films 475, 72, (2005).

[12] J. C. Lee and Y. J. Kim, Vacuum 80, 599 (2006).

[13] P. Chévrier, M. Barrault, C. Fiévet, J. Maftoul, and J. Millon-Frémillon, J. Phys. D Appl. Phys. 30, 1346 (1997).

[14] J. J. Lowke, R. E. Voshall and H. C. Ludwing, J Appl. Physics 44, 3513 (1973)

[15] R. W. Liebermann and J. J. Lowke, JQSRT 16, 253 (1976). 